\title{
LOS JARDINES BOTÁNICOS Y LA CRISIS DE LA BIODIVERSIDAD
}

\author{
Andrew P. Vovides ${ }^{1,3}$, Carlos IGlesias ${ }^{1}$, Víctor Luna ${ }^{1}$ y Teodolinda Balcázar ${ }^{2}$ \\ 'Jardín Botánico "Francisco Javier Clavijero", \\ Instituto de Ecología, A.C., Xalapa, Veracruz, México \\ ${ }^{2}$ Jardín Botánico, Instituto de Biología, Universidad Nacional Autónoma de México México, D.F., México \\ ${ }^{3}$ Autor para la correspondencia: andrew.vovides@inecol.edu.mx
}

\begin{abstract}
Resumen: Históricamente, los jardines botánicos se enfocaban sobre especies con interés económico, y no había mención alguna de la conservación hasta mediados del siglo XX. La destrucción de las selvas y bosques, pérdida de biodiversidad y el cambio climático son problemas reales y los jardines botánicos modernos desarrollaron estrategias para enfrentarlos; la conservación ex situ e in situ, y una alianza de jardínes botánicos para la restauración ecológica. Los jardines botánicos cumplieron ocho de las 16 metas de la Estrategia Global para la Conservación Vegetal, entre ellas, la capacitación de horticultores, que aun hace falta impulsar en México. Se debe fomentar la horticultura a todos niveles, especialmente en la restauración. Los jardines botánicos modernos están repletos de conocimiento sobre la diversidad vegetal, y su acervo de especímenes documentados, respaldados en los herbarios, se utiliza para la sistemática molecular moderna entre otras disciplinas taxonómicamente más tradicionales. Son centros principales para la conservación y han tenido éxito en la educación ambiental. Gracias a las redes nacionales e internacionales, la localización de especies es posible y el concepto de Colecciones Nacionales fue adoptado por jardines botánicos mexicanos. La médula de los jardines botánicos es la calidad de sus bases de datos e información; es urgente reforzar lo anterior entre los jardines de México, incluso utilizar la tecnología del microchip que hace el control de ejemplares más eficiente. Hoy más que nunca, la sociedad necesita el apoyo de los expertos en los jardines botánicos, así como potenciar los mismos como lugares de esparcimiento y tranquilidad.

Palabras clave: alianza de restauración ecológica, colecciones nacionales, colecciones vivas documentadas, conservación ex situ e in situ, horticultura de restauración.
\end{abstract}

\begin{abstract}
Historically, botanic gardens have concentrated on species of economic interest, and no mention of conservation has been made until the mid XX Century. The destruction of forests, loss of biodiversity, and climate change are real problems and modern botanic gardens have developed strategies to confront them; ex situ and in situ conservation and a botanic gardens alliance for ecological restoration. Botanic gardens have addressed eight of the 16 targets of the Global Strategy for Plant Conservation, among these the training of horticulturists. These need to be promoted in Mexico with horticulture courses at all levels, especially in restoration. However, we have succeeded in environmental education. Modern botanic gardens are replete with information on plant diversity, and their holdings of documented specimens include herbarium voucher backup, that are used in modern molecular systematics, among other taxonomically traditional disciplines. Botanic gardens are principal centres of plant conservation and, through national and international networks, specimen location is possible and the concept of national collections has been adopted by Mexican botanic gardens. The backbone of botanic gardens is the quality of databases and information. Reinforcing this is urgent in our gardens, including microchip technology that renders specimen control more efficient. More than ever, does society need the support of experts in botanic gardens as well as spaces for enjoyment and tranquillity.
\end{abstract}

Key words: documented living collections, ecological restoration alliance, ex situ and in situ conservation, national collections, restoration horticulture.

E jardín botánico "oasis de tranquilidad", no nos llega de sorpresa cuando a unos pocos meses después del ataque de las torres gemelas (del 11 de septiembre de 2001), se comentó que los jardines botánicos en los Estados Unidos de América reportaron un incremento notable de visitantes (Powledge, 2011). Hoy más que nunca, los jardines botáni-

cos han adquirido una gran importancia como espacios para tranquilidad y esparcimiento para los habitantes de las grandes ciudades, cada día más alejados de la naturaleza. Estas islas de tranquilidad y serenidad, tratan de reconciliar a la humanidad con la naturaleza y sostener al espíritu humano por medio de la exposición de las plantas en toda su mag- 
nificencia y hermosura; asimismo, proporcionar vías para la investigación y la educación. A nivel mundial, los jardines botánicos reciben alrededor de 250 millones de visitantes al año y cultivan unas 115,000 especies (Wyse Jackson, com. pers.). Sin embargo, atrás de los paisajes y camellones idílicos de árboles, flores y estanques con nenúfares, existen edificios y laboratorios llenos de científicos enfrascados en sus tareas de describir y revelar los secretos del mundo vegetal. Desde siglos atrás, los jardines botánicos fungían y siguen fungiendo como repositorios de plantas y conocimientos botánicos. Ya en el México precolombino, los jardines botánicos estaban plenamente desarrollados cuando apenas se estaban iniciando en Europa, aunque no fueron estrictamente jardines botánicos en el sentido moderno. El conocimiento botánico de los antiguos mexicanos fue influenciado por su cosmovisión religiosa y mística con un gran conocimiento de especies medicinales (Valdés, 1974; León-Portilla, 1979; Lascurain, 2010). Un ejemplo es el jardín de Oaxtepec (Durán, 1967), que entre otras plantas en sus colecciones, se destacan las medicinales, las cuales probablemente fueron usadas por el Hospital de La Santa Cruz de Oaxtepec, fundado por lo hermanos Hipólitos en 1556 (Miranda, 1960). Otro jardín precolombino es el del rey Netzahualcóyotl en el bosque de Tecotzinco, donde el riego de las plantas se realizaba por acueductos (Lascurain, 2010).

Durante el período colonial europeo las metas prioritarias en los jardines se enfocaban sobre especies con interés económico, para su introducción e intercambio, además de ser repositorios de la flora exótica de las colonias europeas para su estudio taxonómico, así como contribuir al incremento de los acervos de los herbarios adjuntos y la riqueza hortícola y de jardinería de estos países (Moore, 1974; Bye, 1994). Hoy en día nos percatamos que nunca antes en la historia han sido tan importantes los jardines botánicos en la investigación científica (Crane et al., 2009).

\section{Jardines botánicos y la conservación}

En los jardines botánicos no había mención alguna de la conservación hasta mediados del siglo XX (Prance, 2010), y en la actualidad, estas instituciones han evolucionado en sus metas para enfrentarse con la problemática de la pérdida de biodiversidad, especies amenazadas y en peligro de extinción, especies invasoras, conservación ex situ e in situ, el calentamiento global y recientemente, la restauración ecológica. Cabe mencionar que más de la mitad de los 2,500 principales jardines botánicos del mundo fueron establecidos después de 1950 (Crane et al., 2009), y ahora existen más de 3,000 (Wyse Jackson com. pers). Comenta Peter Raven, ex director del Missouri Botanical Garden que "los jardineros pueden tener un papel clave en los esfuerzos para proteger la biodiversidad" (Marris, 2006).

La mayoría de los jardines botánicos modernos incluyen en su misión un compromiso para conservar la diversidad vegetal y enfrentar la extinción y pérdida de biodiversidad. Los ambientes naturales y la diversidad están siendo destruidos, desaparecen bajo la expansión y desarrollo de actividades agropecuarias, minería, explotaciones petroleras, comercio, expansión urbana, sobrecolecta de especies carismáticas y medicinales, entre otras. La destrucción y quema de las selvas y bosques aporta una quinta parte de las emisiones de carbono en la atmósfera, más de lo que producen los sistemas de transporte a nivel mundial (Crane et al., 2009). El cambio climático está afectando la permanencia de ciertas especies en sus áreas naturales y, en algunos casos, causa su desaparición o migración; así como la mortandad masiva de las especies en los bosques debido al estrés hídrico (Choat et al., 2012); además, la alteración de los climas tendrá impactos serios sobre los ecosistemas y las poblaciones humanas (Walther et al., 2002; Thuiller et al., 2005; Cushing y Kopas, 2011). Hoy en día, los jardines botánicos están adoptando políticas y respondiendo a las necesidades globales como el alivio de la pobreza, la seguridad alimenticia, la sustentabilidad, el cambio climático y los problemas de especies invasoras, entre otras.

Los botánicos han reconocido que los problemas de extinción, pérdida de biodiversidad y cambio climático son problemas reales, y el manto vegetal del planeta requiere de nuestra atención urgente (Heslop-Harrison, 1976; Raven, 1976), por lo que han establecido estrategias para enfrentarlos (Wyse Jackson y Sutherland, 2000; BGCI, 2006). En México en particular, los problemas antes mencionados, e incluso el uso y acceso de los recursos genéticos, se plantean y se discuten en la "Declaración Oaxaca" (De Ávila et al., 2003) y en "La Estrategia de Conservación para los Jardines Botánicos Mexicanos" (Rodríguez-Acosta, 2000). Se recomienda la vinculación estrecha entre los jardines botánicos y las autoridades federales, estatales y municipales, ONGs y comunidades; así como el establecimiento de jardines botánicos en áreas con alta diversidad biológica y endemismos. En México, una gran proporción de la biodiversidad se encuentra en tierras comunales (Maunder, 2006), por lo tanto, se hace más urgente el establecimiento en áreas comunales, tanto de jardines botánicos como de unidades de manejo y aprovechamiento (UMA) como una estrategia alterna a la conservación con miras a la sustentabilidad; un ejemplo de esto es el Jardín Botánico "Helia Bravo Hollis" de Zapotitlán de las Salinas, Puebla, situado en el Valle de Tehuacán, área de alto endemismo de cactáceas (Vovides et al., 2002, 2010a). El Jardín Botánico "Fco. J. Clavijero" ha asesorado UMAs para cícadas desde 1990 (Vovides et al., 2010b). El propósito de las UMA es crear un incentivo a conservar las especies silvestres carismáticas y su hábitat por el uso sostenido mediante un plan de manejo que incluya propagación, venta de plantas y reforestación. Los planes de manejo de las UMA cuentan con la asesoría y seguimiento de expertos que a menudo, son personal de los jardines botánicos. 
Conservación ex situ. La conservación ex situ se considera deficiente, debido a que no es posible mantener más que una muestra limitada de la diversidad genética de las especies, que a la larga puede dar cambios impredecibles y convertirse en domesticación; por lo tanto, la conservación in situ es preferible (Raven, 1976). La conservación in situ, permite que las poblaciones se desarrollen y se relacionen dentro de sus hábitats naturales. Sin embargo, en el mundo real y en la práctica, los dos métodos son necesarios dado que la conservación ex situ actúa como respaldo para ciertos segmentos de la diversidad que podrían desaparecer en la naturaleza y en los ecosistemas dominados por la humanidad (Cohen et al., 1991; Wyse Jackson y Sutherland, 2000). Los jardines botánicos son quizás las únicas instituciones que cuentan con infraestructura y personal especializado con la experiencia en taxonomía, horticultura y especialmente, adaptados para el manejo de especies vivas en colecciones que jamás han sido cultivadas ni domesticadas. La horticultura es un componente esencial en programas de conservación vegetal y, el personal de los jardines botánicos puede asesorar y dirigir acciones de reintroducción y restauración ecológica en colaboración con los ecólogos especialistas de las reservas de la biósfera, áreas naturales protegidas y otras áreas de interés. Las plantas cultivadas en los jardines botánicos son una fuente importante para acrecentar las poblaciones silvestres pequeñas de especies endémicas o en peligro crítico de extinción, especialmente las panmícticas con poca diferenciación genética, incluso, en algunos casos, con el uso de ejemplares no documentados (Da Silva et al., 2012). Existe gran potencial en los jardines botánicos para utilizar el mercado para avanzar en la horticultura conservacionista; algunos mantienen bancos de semillas como el Millenium Seed Bank de Kew, Inglaterra, otros como el Montgomery Botanical Center de Florida, EUA, dan énfasis a la propagación y distribución de semillas y polen de especies carismáticas como las cícadas y las palmas. Un ejemplo de esto, es la propagación de la "palma corcho" (Microcycas calocoma (Miq.) A.DC.), endémica de Cuba y críticamente en peligro de extinción, que reduce la presión de colecta ilegal en su hábitat (Kay et al., 2011).

\section{Restauración ecológica}

Hardwick et al. (2011) recomiendan que los jardines botánicos incluyan a los ecosistemas en sus investigaciones, incrementen su participación en proyectos prácticos de restauración y en la capacitación de recursos humanos, y además, sean centros de información e intercambio de datos. Sostienen que una reorientación de la potencialidad existente en los jardines botánicos, como la investigación botánica, la horticultura y la transferencia de conocimiento, permitirán que muchos jardines botánicos alrededor del mundo proporcionen un apoyo efectivo a los esfuerzos de restauración ecológica. Sin duda alguna, de los jardines botánicos emer- gerá una nueva disciplina híbrida entre la ecología vegetal, la taxonomía y la horticultura, formando así, un nuevo profesional en la horticultura de restauración.

Alianza de jardines botánicos para la restauración ecológica. La Sociedad para la Restauración Ecológica (SER, por sus siglas en inglés), en su $4^{\mathrm{a}}$ conferencia mundial del 2011 en Mérida, Yucatán, emitió recomendaciones en torno a la restauración ecológica, entre las cuales destacan: "Adoptar guías para establecer prioridades y metas para las actividades de restauración, y facilitar la transferencia de conocimientos, herramientas y tecnologías". Al mismo tiempo se hizo un llamado para solicitar más apoyo del Banco Mundial, El Fondo para el Medio Ambiente Mundial y otras agencias (SER, 2011). La Botanic Gardens Conservation International (BGCI) convocó la formación de una alianza de jardines botánicos en el año 2010 para la restauración ecológica, que incluye 100 jardines botánicos a nivel mundial que tienen terrenos con vegetación nativa. La misma incluye jardines botánicos in situ en donde se enfocan en la conservación in situ y restauración en los trópicos (Crane et al., 2009; Vovides et al., 2010a). Esta nueva alianza se dedicará a impulsar un esfuerzo mundial con grandes metas a largo plazo, con el propósito de entregar resultados en torno a la restauración, en apoyo al Convenio de la Diversidad Biológica (CDB), al marco de la Convención sobre el Cambio Climático y otros acuerdos internacionales. Esto se realiza con base en el conocimiento de la flora nativa y la vegetación, y el reconocimiento de que los jardines botánicos juegan un papel científico crítico en el estudio, entendimiento y manejo de muchos ecosistemas locales; además, se busca una relación beneficiosa con la SER. Varios jardines botánicos mexicanos que se construyeron en ecosistemas nativos califican para esta acción de conservación y algunos ejemplos son: el Jardín Botánico "Francisco Javier Clavijero" del Instituto de Ecología, A.C. (INECOL) con el Santuario del Bosque de Niebla y, el recientemente adquirido terreno en el año 2012, con un área de bosque de niebla o mesófilo y humedal que estaban bajo amenaza de urbanización; el "Jardín Botánico del Instituto de Biología de la Universidad Nacional Autónoma de México" (UNAM), con experiencias de reintroducción y conservación de especies amenazadas de la Reserva Ecológica del Pedregal de San Ángel de la Ciudad Universitaria (Rubluo et al., 1989); el Jardín Botánico "Dr. Alfredo Barrera Marín" del Colegio Frontera Sur, en Puerto Morelos, Quintana Roo, con remanentes de selva tropical y manglar del Caribe mexicano; el Jardín Botánico "Helia Bravo Hollis" de Zapotitlán Salinas, Puebla, situado en un área de gran endemismo de plantas xerófitas y cactáceas; entre otros.

\section{Los jardines botánicos y la sistemática}

Taxónomos "en peligro de extinción". Los taxónomos clásicos de plantas; es decir, los que basan su trabajo fundamentalmente en la morfología, son cada día más escasos dado 
que se están jubilando y no están siendo reemplazados, ya que sus plazas se ocupan particularmente con investigadores especializados en sistemática molecular (Campbell, 2006; Marris, 2006), lo cual resultará a largo plazo en un desequilibrio no deseado en la botánica. Los profesionales tradicionales que consideran al organismo como un todo se están convirtiendo rápidamente en una "cultura en desaparición", un hecho especialmente más grave en Latinoamérica, donde aún no se conoce a fondo su biodiversidad vegetal, y la misma está siendo mermada a un paso acelerado por las actividades humanas. Algunos botánicos se preocupan porque los apoyos limitados disponibles se dan a otros proyectos "prioritarios", en lugar de aplicarse en los jardines botánicos para avanzar el conocimiento de las floras locales, regionales y promover su conservación y rescate.

Un jardín botánico está repleto de conocimiento sobre la diversidad vegetal, y su acervo de plantas vivas documentadas es un laboratorio ideal para la investigación moderna, gracias a los horticultores que mantienen tales colecciones vivas. Para los sistemáticos, estas colecciones son útiles para detectar, por medio de métodos moleculares y morfología tradicional, complejos de criptoespecies. Es decir, especies aisladas en su reproducción pero similares en morfología, a veces indistinguibles. Mark Chase, sistemático molecular de Kew, lo llama "iluminación recíproca", en donde se mira a la planta entera, luego a las moléculas y después a la planta, y menciona: "realmente ves cosas que no te has dado cuenta previamente" (Marris, 2006).

Depositarios de los herbarios. Hoy día los jardines botánicos han cobrado aún mayor importancia, ya que incluyen ejemplares botánicos vivos que tienen respaldo en los herbarios y que se utilizan para la investigación molecular. La extracción de ADN de los ejemplares vivos de las colecciones tiene un gran valor, dado que reduce o elimina el trabajo de campo. Por otro lado, es mucho más fácil la extracción del ADN de material fresco que de ejemplares herborizados. Por lo tanto, la sistemática molecular, junto con otras disciplinas tradicionales, forman parte de la misión de los jardines botánicos modernos, en sus esfuerzos de entender la biología de las plantas. También en los jardines botánicos se apoya la conservación en tres maneras importantes: (1) se estudia la diversidad vegetal y se establecen cuáles son las especies amenazadas o raras (Marris, 2006), (2) se generan protocolos de germinación y de cultivo de especies silvestres poco conocidas con miras a la reintroducción y restauración (Bramwell, 1991) y (3) se difunden los conocimientos por medio de educación, difusión y exposiciones (Linares et al., 2006; Vovides et al., 2010a).

\section{Educación ambiental}

Ceguera vegetal. Más que nunca, la sociedad necesita el apoyo de los expertos que trabajan en los jardines botáni- cos; sin embargo, muy a menudo estas instituciones son escasas y carentes de fondos suficientes, principalmente por la denominada "ceguera vegetal" que la sociedad ha ido arrastrando desde hace muchos siglos (Powledge, 2011). La ceguera vegetal es un término introducido por los profesores James Wandersee y Elizabeth Schussler citados por Powledge (2011) que se refiere a: "la inhabilidad de los humanos de ver o darse cuenta de las plantas en su ambiente; la incapacidad de reconocer la importancia de las plantas en la biósfera y en la vida cotidiana de los humanos, así como la categorización descarriada y antropocéntrica de las plantas como inferiores a los animales y, así, no merecedores de consideración." Es cierto, sí uno pregunta a un grupo de niños qué prefieren visitar, a un jardín botánico o un zoológico, seguramente la respuesta de la mayoría sería visitar el zoológico, o mejor aún, ir al cine. Los humanos fácilmente nos identificamos con los animales y los consideramos más importantes que las plantas. El remedio para tal padecimiento de la "ceguera vegetal" es la educación botánica y tutoría, y la experiencia directa para hacer evidente y valorar el mundo vegetal. Los jardines botánicos son particularmente útiles para esta tarea desafiante y muchos de ellos tienen programas de educación ambiental ambiciosos.

El Fairchild Challenge. Uno de los objetivos más importantes, y de gran éxito para la educación ambiental en los jardines botánicos, se ha conseguido por medio del denominado programa Fairchild Challenge (FC), o sea, el reto Fairchild. Es un concepto desarrollado por investigadores del Fairchild Tropical Botanic Garden en Miami, Florida, que involucra a maestros y alumnos de las escuelas primarias, secundarias y preparatorias en donde investigan y evalúan críticamente temas de relevancia ambientales, y así puedan convertirse en ciudadanos más participativos para llegar a apreciar de lleno el valor de la naturaleza. Esto se lleva a cabo mediante proyectos llamados "desafíos", de los cuales los alumnos junto con sus maestros los desarrollan, y al final participan en un concurso que premia los mejores proyectos.

Este programa ha tenido mucho éxito en los EUA e internacionalmente, y se está implementando en México por primera vez de manera formal en el Jardín Botánico "Francisco Javier Clavijero" (JBC) del Instituto de Ecología, A.C. (INECOL) en Xalapa, en colaboración con la Secretaría de Educación de Veracruz; los cuales, además, son pioneros en la historia del FC y el JBC con respecto a la participación de alumnos discapacitados. Han tomado parte en este proyecto casi 300 maestros con más de 3,000 alumnos, y en el segundo ciclo (2012-2013) 180 escuelas y 7,000 niños.

\section{Las redes de jardines botánicos}

La Asociación Mexicana de Jardines Botánicos. A partir de la creación de la Asociación Mexicana de Jardines Botánicos, A.C. (AMJB), en 1983, con la primera Reunión Nacio- 
nal en 1985, se inició una comunicación eficiente entre los jardines botánicos, que resultó en la eliminación de previos aislamientos interinstitucionales. Anteriormente, los encargados de los jardines botánicos únicamente se reunían durante los congresos de botánica trienales de la Sociedad Botánica de México. Hoy en día, entre los aproximadamente 40 jardines botánicos adscritos a la AMJB se mantiene una estrecha y activa comunicación mediante reuniones regionales y nacionales así como con el boletín informativo Amaranto. Además de la publicación de un directorio de jardines botánicos (Herrera et al., 1993), la página web y la existencia de publicaciones especiales con relación a los quehaceres en los jardines botánicos, que van desde la educación ambiental hasta sistemas de registro y otros temas de conservación, mantenimiento de colecciones vivas y asuntos de curatoría, todo esto reforzado por talleres periódicos y reuniones especiales.

México fue el primer país latinoamericano en formar una Red Nacional de Jardines Botánicos, seguido por Cuba en 1986 y luego Argentina en 1987. En los años 80 y 90, el crecimiento en número de jardines botánicos en México fue extraordinario, desde menos de cuatro en los años 50 hasta treinta y cinco adscritos a la AMJB a finales de 1989. Las actividades cubiertas por la mayoría de ellos son taxonomía, propagación y etnobotánica y, en menor grado, las disciplinas como ecología, horticultura, cultivo de tejidos, biosistemática, genética, entre otras (Vovides et al., 2010a). Desde 1990 al presente, la creación de jardines botánicos en nuestro país continua creciendo, por lo menos diez jardines más adscritos a la AMJB, y en el 2012, de los 32 estados de la República Mexicana, solamente, Chihuahua, Colima, Nayarit, San Luis Potosí, Sonora y Zacatecas no cuentan con jardines botánicos adscritos la Asociación (Figura 1).

La distribución de los jardines botánicos en México se encuentra concentrada, en su mayoría, en los estados del centro del país, donde 21 jardines botánicos adscritos a la Asociación, con diversas colecciones, contribuyen de manera significativa a la conservación de la diversidad vegetal.

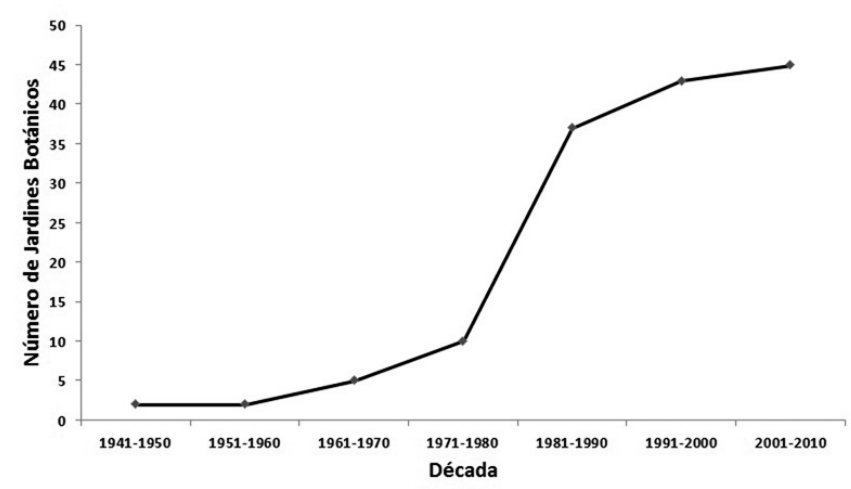

Figura 1. Fundación de jardines botánicos en México adscritos a la Asociación Mexicana de Jardines Botánicos, A.C. (1941-2010).
Muy probablemente existen más jardines botánicos, pero no se tiene datos de ellos. Sin embargo, la permanencia de los jardines botánicos es fluctuante y uno de los principales problemas que presentan, es su desaparición por falta de apoyo económico o del reconocimiento del valor de las colecciones por las autoridades (¿la "ceguera vegetal?”). Esto se da, en parte, debido a que en México los jardines botánicos pertenecen a alguna institución pública de investigación, colegio o universidad, sujetos a las políticas cambiantes que no siempre están a favor del mantenimiento de colecciones. Sería ideal que estos jardines botánicos sean instituciones en sí mismas con sus propios rumbos y políticas, como las grandes instituciones extranjeras: el Jardín Botánico de Río de Janeiro, Brasil: el Missouri Botanical Garden y el New York Botanical Garden de los EUA; el National Botanic Gardens de Dublin, Irlanda: el Royal Botanic Gardens de Kew del Reino Unido; Royal Botanic Garden Edinburgh de Escocia y el Royal Botanic Gardens en Sidney, Australia, por mencionar algunos.

La Botanic Gardens Conservation International y las metas del Global Strategy for Plant Conservation. Tanto la mayoría de los jardines botánicos nacionales como del extranjero, mantienen contacto estrecho con la Botanic Gardens Conservation International (BGCI por sus siglas en inglés). La colaboración del BGCI con el Convenio de la Diversidad Biológica da las Naciones Unidas (CDB) han contribuido al éxito de la implementación de algunas de las metas de la Estrategia Global para la Conservación Vegetal (GSPC, por sus siglas en inglés). La primera fase de esta estrategia fue llevada a cabo entre 2002 al 2010 y fue aceptada ampliamente por los jardines botánicos. El CDB adoptó una segunda fase de la GSPC que incorpora metas revisadas para cumplirse en 2020. En la primera fase de la GSPC se alcanzaron numerosos éxitos con avances sustanciales para ocho de las dieciséis metas al 2010, a pesar de no alcanzar el objetivo principal de detener la pérdida de la diversidad vegetal (Blackmore et al., 2011). Las ocho metas según Rae (2004), donde más han contribuido los jardines botánicos son:

1. Meta Uno: la creación de una lista de las especies conocidas, ampliamente accesible como un paso hacía una flora mundial. Aquí las colecciones de los jardines botánicos han apoyado tradicionalmente y contribuido a la investigación taxonómica, principalmente en el cultivo de especies difíciles de cultivar, hasta lograr la floración y fructificación, y así completar su descripción taxonómica. Muchos jardines cuentan con una base de datos centralizada de sus colecciones, así como contribuciones a la base de datos de la BGCI. Sin embargo, en México, un buen número de jardines botánicos requieren reforzar la importancia de los registros de accesiones y documentación de sus colecciones.

2. Meta Tres: el desarrollo de modelos con protocolos para la conservación y uso sustentable, basados en investigación y experiencia práctica. Esta meta hace énfasis sobre la 
importancia de la horticultura en el éxito de programas de conservación, un ejemplo es el Programa Internacional de Conservación de las Coníferas en el Jardín Botánico Real de Edimburgo (Rae, 2004). Para México, se puede mencionar el desarrollo de ocho colecciones nacionales, mencionadas con detalle más adelante, y la implementación del concepto de unidades de manejo y aprovechamiento integral de la flora y fauna (UMA) de SEMARNAT para la propagación y conservación de cactáceas y cícadas, entre otras especies ornamentales con miras a la sustentabilidad.

3. Meta Siete: el 60\% de las especies amenazadas del mundo deben ser conservadas in situ. Dada la presión de deterioro sobre las reservas ecológicas y otras áreas naturales protegidas por actividades humanas, de una manera u otra necesitan cierta intervención y manejo, y no se debe menospreciar el papel de los jardines botánicos como asesores para "ayudar" a las especies con problemas de sobrevivencia.

4. Meta Ocho: estipula que el $60 \%$ de las especies amenazadas estuviesen en colecciones ex situ, especialmente en su país de origen, y el 10\% incluidas en programas de rescate y restauración. Aquí el papel de los jardines botánicos es muy importante para alcanzar esta meta, al ser efectivos en la propagación y cultivo de especies amenazadas (Blackmore et al., 2011). En el caso de las cícadas, casi el $90 \%$ de las 330 especies conocidas a nivel mundial (Osborne et al., 2012) están representadas en colecciones vivas en los jardines botánicos. 5. Meta Diez: las especies invasoras están cobrando más atención hoy día, debido a que causan estragos en ecosistemas frágiles. La identificación y control de las especies invasoras involucra técnicas de taxonomía y horticultura; así como la vigilancia de parte de los jardines botánicos para que estas especies no se escapen del cultivo y asegurar áreas propensas a invasiones.

6. Meta Catorce: el papel de la comunicación acerca de la importancia de la diversidad vegetal y su conservación. $\mathrm{La}$ mayoría de los jardines botánicos cuentan con profesionales en la educación y difusión, pero esta tarea no se debe limitar a los docentes. También los horticultores y taxónomos son buenos comunicadores y la sinergia entre las profesiones suele ser muy positiva. En México existen varios programas de educación ambiental y talleres entre el $80 \%$ de los jardines botánicos mexicanos (Vovides et al., 2010a), algunos ejemplos son los Jardines Botánicos "Dr. Alfredo Barrera Marín" de Puerto Morelos, Quintara Roo; el Etnobotánico del INAH, Cuernavaca, Morelos; el Entobotánico de la ciudad de Oaxaca; el Fco. J. Clavijero del INECOL, Xalapa, Veracruz; del Instituto de Biología, UNAM, y el Xiítbal Neek del CICY, Mérida, Yucatán.

7. Meta Quince: la construcción de capacidades para la conservación, en donde el número de personal capacitado y trabajando en la conservación de plantas debe incrementarse según las necesidades nacionales. En el Reino Unido, por medio de la Iniciativa Darwin, se ha promovido el intercambio de personal entre el Jardín Botánico de Edimburgo y el Jardín Botánico Serbitang en Bhutan. De esta manera, personal de ambos jardines se han beneficiado del intercambio (Rae, 2004). En México, aún hace falta impulsar esta modalidad y se debe fomentar y apoyar cursos en horticultura, desde certificación de jardineros y diplomados hasta posgrados. El concepto de "jardines botánicos hermanos" tiene potencial para implementar esta meta por medio de intercambio de personal docente en horticultura.

8. Meta Dieciseis: la creación de redes para actividades de conservación a niveles nacional, regional e internacional. Las redes son excelentes oportunidades en las que el personal de los jardines botánicos se reúne para compartir ideas, técnicas y soluciones a problemas. Estas ofrecen distintas oportunidades para organizar talleres, conferencias y proyectos en conjunto, así como colaboraciones con otras instancias gubernamentales y ONGs. En México esto ocurre entre los jardines miembros de la AMJB a nivel nacional y regional, y con la BGCI a nivel internacional. Sin embargo, para tener más éxito, se requiere el mejor entendimiento y la coordinación de los jardines botánicos, así como el reforzamiento de su investigación científica, de las normativas y los compromisos institucionales y gubernamentales, además del papel de la horticultura en los mismos, sin dejar a un lado la formación de personal y la colaboración internacional (Rae, 2004; Blackmore et al., 2011).

Localización de especies. Muchos investigadores, para fines de investigación, ya aprovechan las colecciones vivas de los jardines botánicos; y en casos en los que no está disponible el taxon de interés, la BGCI ofrece un servicio de localización de especies de plantas cultivadas en otros jardines botánicos del mundo por medio de su sitio de Internet Plant Search (http://www.bgci.org/plant_search.php), para intercambio científico. En ese sitio, el investigador puede efectuar una búsqueda del taxon requerido en línea y, la BGCI, con su base de datos de jardines botánicos adscritos, contacta el jardín o jardines en donde está disponible la especie de interés; de esta manera, el jardín donante a su vez contacta al investigador para algún posible intercambio. Si los taxa están listados en la NOM Oficial o CITES, entonces es necesario el trámite de los premisos respectivos para su exportación o importación como intercambio científico. La Comisión Nacional para el Conocimiento y Uso de la Biodiversidad (CONABIO), en México cuenta con la Red Mundial de Información sobre la Biodiversidad (REMIB), que proporciona información para consultas de taxa en donde tienen registradas 33 instituciones nacionales y extranjeras, algunas de ellas jardines botánicos.

\section{Las colecciones nacionales en jardines botánicos y do- cumentación}

Historia. Las Colecciones Nacionales históricas en México consisten de las colecciones herborizadas y zoológicas 
del Instituto de Biología de la UNAM, decretadas como Nacionales por el Presidente Emilio Portes Gill en 1929 (IB-UNAM). El concepto moderno de las colecciones nacionales de plantas nació en la Gran Bretaña, desarrollado por el Consejo Nacional para la Conservación de Plantas y Jardines (NCCPG, por sus siglas en inglés), fundado en al año 1978. El objetivo del NCCPG es combinar los talentos de botánicos, de horticultores y de conservacionistas, junto con la experiencia de jardineros tanto profesionales como aficionados. La misión de este Consejo es conservar, cultivar, propagar, documentar y hacer disponible al público el asombroso recurso que representan las plantas cultivadas y amenazadas en el Reino Unido, incluyendo los jardines privados, públicos destacados e históricos. Asimismo, tienen como objetivo promover la propagación, conservación y fomentar la investigación sobre plantas cultivadas, sus orígenes, ambientes, así como su importancia histórica y cultural. Igualmente tratan de fomentar la educación del público general en torno a la conservación de plantas de jardín y de jardines históricos. La conservación de plantas cultivadas ha sido tomada muy en serio, pues se ha dicho que "las [plantas] favoritas de ayer son las extintas de mañana", de tal forma que se han publicado directorios de dichas colecciones y jardines (NCCPG, 2011).

Conservación de variedades en desuso. A nivel mundial se reconoce la importancia de conservar las variedades antiguas de plantas no comerciales y los parientes silvestres de los cultivos para el fitomejoramiento, la resistencia a plagas y condiciones ambientales adversas (Heslop-Harrison, 1974; Cohen et al., 1991). Por ejemplo, existen 7,500 variedades de manzanas (Malus domestica $\mathrm{L}$.) a nivel mundial (University of Illinois); sin embargo, en los supermercados únicamente están disponibles unas cuantas. ¿Quién se preocupa por las demás variedades? En el caso de México, existe el Sistema Nacional de Recursos Fitogenéticos para

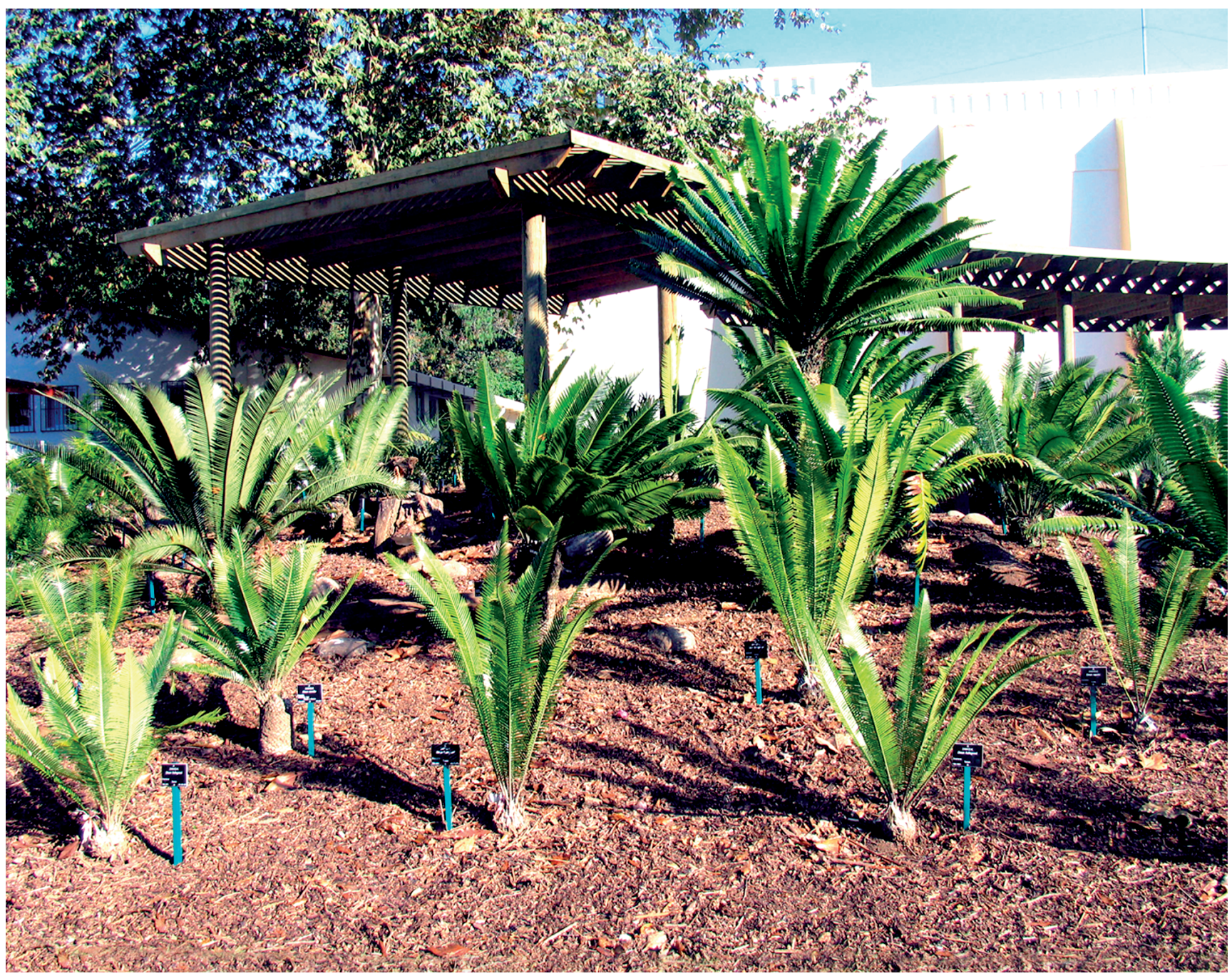

Figura 2. Colección Nacional de Cícadas, Jardín Botánico Fco. J. Clavijero, Instituto de Ecología A.C. 


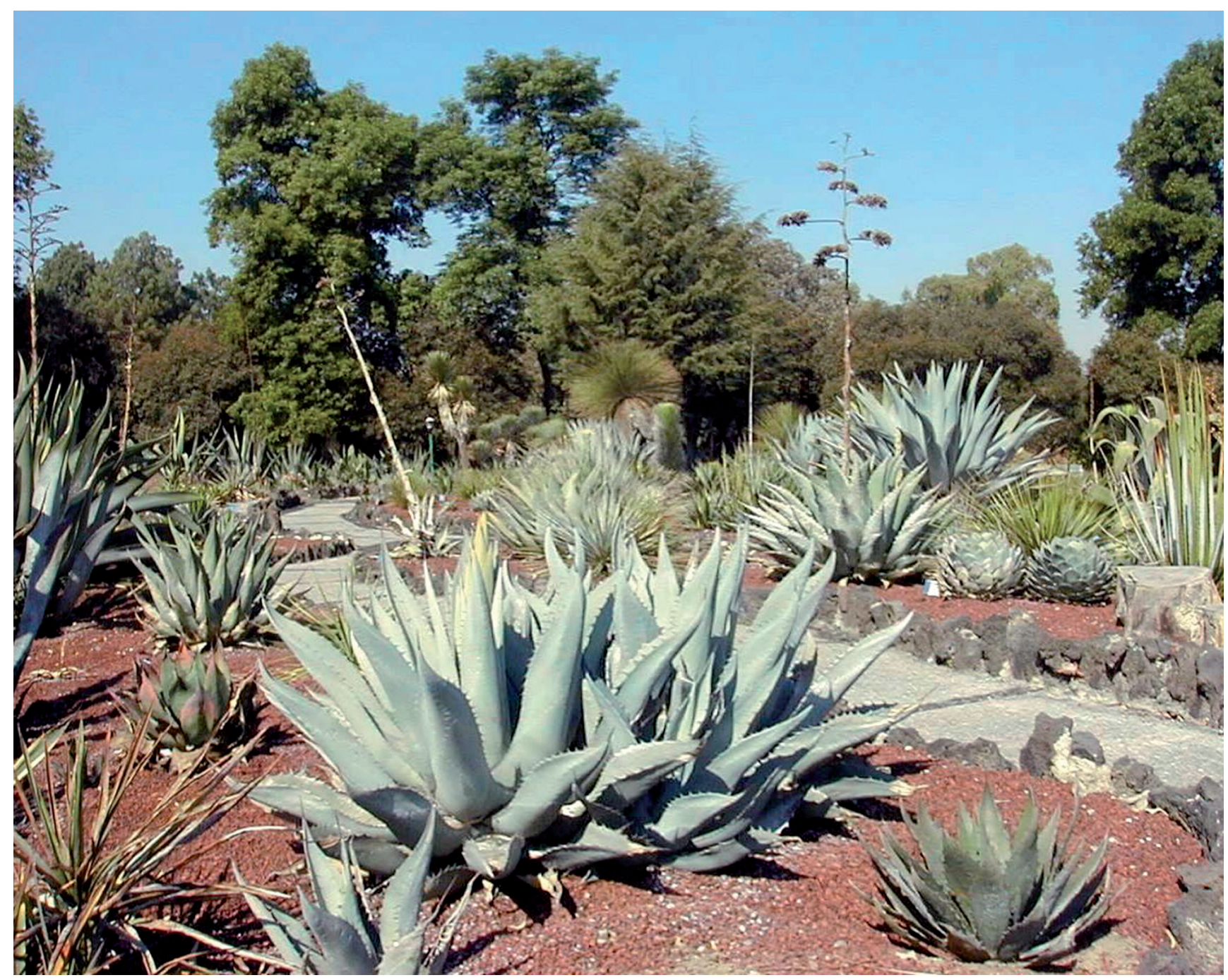

Figura 3. Colección Nacional de Agaváceas, Jardín Botánico Instituto de Biología, UNAM.

la Alimentación y la Agricultura (SINAREFI), que cuenta dentro de sus programas con la conservación de las especies emparentadas y silvestres de los cultivos (Cuevas-Sánchez y Muñoz, 2006). Sin embargo, según la diagnosis de Molina-Moreno y Córdoba-Tellez (2006), la conservación ex situ de semillas no es óptima. Con la creación, en el año 2012, del Centro Nacional de Recursos Genéticos (CNRG), que utiliza tecnología de vanguardia, se pueden resolver algunos problemas de almacenamiento de germoplasma. El Banco Nacional de Germoplasma Vegetal (BANGEV), en Chapingo, Estado de México, cuenta con más de 19,000 accesiones; entre ellas, plantas vivas en el Jardín Agrobotánico del Centro Regional de la Península de Yucatán de la Universidad Autónoma de Chapingo. No obstante, los jardines botánicos regionales y arboretos juegan un papel importante en la conservación ex situ de variedades en desuso o incluso, abandonadas para la posteridad, y otras de uso muy local o regional, no consideradas por los bancos de germoplas- ma; p.ej. el cachichín (Oecopetalum mexicanum Greenm. \& C.H.Thomps.) de la región de Misantla, Ver. y el tepetomate (Pseudolmedia glabrata (Liebm.) C.C.Berg) del sur y sureste mexicano (Lascurain et al., 2010). Los grandes bancos de germoplasma, como el Consejo Internacional para Recursos Genéticos Vegetales (IBPGR, por sus siglas en inglés), se ocupan únicamente de las variedades cultivadas. Para las especies silvestres, son los jardines botánicos los centros principales para su conservación (Heywood, 1991).

Colecciones nacionales en jardines botánicos en México. Mientras en la NCCPG se estableció el concepto de colección nacional para jardines y especies domesticadas, en México nació la idea de extender este concepto, entre los jardines botánicos, a las especies de plantas mexicanas silvestres y amenazadas. En los años ochenta, durante los años formativos de la AMJB, se adoptó este concepto y se decidió extenderlo a las especies de plantas mexicanas sil- 


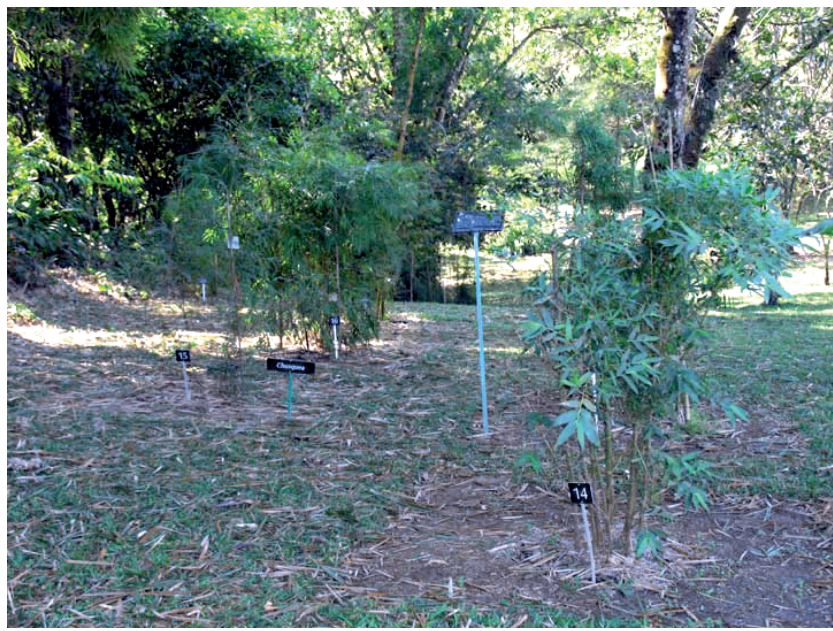

Figura 4. Colección Nacional de Bambúes, Jardín Botánico Fco. J. Clavijero, Instituto de Ecología A.C.

vestres. La primera colección nacional en establecerse fue la de las cícadas del Jardín Botánico Fco. J. Clavijero (Vergara Silva et al., 2002; Figura 2). Hoy en día, en los jardines botánicos de México existen por lo menos ocho colecciones de este tipo, algunas consolidadas y otras en desarrollo. Ejemplos de ellas son las Colecciones Nacionales de Agaváceas y Nolináceas del Jardín Botánico del Instituto de Biología de la UNAM (García-Mendoza, 2007), donde se encuentran representadas 119 especies de las 330 existentes para la familia Agavaceae. Los géneros representados son Agave, Baschomeria, Furcraea y Manfreda; el género Agave predomina en la colección con 80 especies (Figura 3). En el 2010 se estableció la Colección Nacional de Nopales Silvestres, en ella se encuentran 67 de las 103 especies de nopales silvestres conocidos en México. Además, la Colección Nacional de Crasuláceas, reconocida en el año del 2005, por incluir más del 70\% de las especies mexicanas. La Colección Nacional de Encinos del Jardín Botánico "Ignacio Rodríguez Alconedo", de la Benemérita Universidad Autónoma de Puebla, enfocada en una colección de encinos regionales de Puebla y de regiones similares, de los cuales tiene el 32\% del total registrados para México (Rodríguez-Acosta et al., 2008). La Colección Nacional de Plantas Medicinales del Jardín Etnobotánico del INAH, en Cuernavaca. La Colección Nacional de Bambúes Nativos de México del JBC (Figura 4), que consiste de 25 especies de las 36 registradas en México, con las cuales se realizó un proyecto de códigos de barras genéticos (Sosa et al., 2013), una filogenia molecular del género mesoamericano Olmeca (Ruíz-Sánchez et al., 2011a) y la revisión del género Otatea (Ruíz-Sánchez et al., 2011b); la Colección Nacional de Cícadas del mismo jardín, donde se tiene desde 1 a 11 especies de los diez géneros representativos del orden Cycadales, y las 55 especies conocidas de la familia Zamiaceae nativas de México. Con base en esta colección se han realizado nu- merosos trabajos taxonómicos, sistemáticos (Vovides et al., 2007), y basados en la colección se han descrito 20 especies nuevas para el país y la ciencia.

Documentación. Una de las grandes fortalezas de las colecciones científicas en los jardines botánicos es la calidad de las bases de datos de las mismas, con información taxonómica y procedencia de los ejemplares disponibles a consulta por investigadores u otras personas (Sosa, 2004; Luna, 2006). Sin la documentación adecuada de sus ejemplares, el jardín botánico se vuelve sencillamente un jardín o parque, no importa lo interesante y diverso que puedan ser en sus colecciones. Las bases de datos que se manejan van desde libros de acceso tipo contables o tarjeteros hasta métodos computarizados. Como cualquier colección científica, cada ejemplar tiene asignado un número de registro o ingreso respaldado por una ficha en la base de datos del jardín con toda la información relacionada al ejemplar, como los datos de interés hortícola. Cada planta viva colectada debe tener respaldo de ejemplar de herbario, y posteriormente datos sobre su ubicación y seguimiento hortícola en el jardín botánico (Leadlay y Greene, 1998). La ubicación dentro del jardín es apoyada por sistemas de mapas en camellones o en los invernaderos, así como información contenida en una base de datos sobre su propagación, condiciones de cultivo, enfermedades, bajas y donaciones. El seguimiento de cada ejemplar utilizado en proyectos de investigación tiene como objetivo el crear un historial sobre cada ejemplar, y así incrementar su valor científico; por ejemplo, extracción de ADN, tejidos y órganos para investigaciones diversas. La utilidad de esta última información es enorme; los datos de investigaciones taxonómicas, filogenéticas, citológicas o anatómicas basada en esos ejemplares, estarán disponibles para enriquecer trabajos posteriores sobre el mismo ejemplar. En la actualidad todo esta información se maneja por bases de datos electrónicas, tales como la BG-Base ${ }^{\circledR}$, los cuales están respaldadas por sistemas de seguimiento geográfico como BG-Map ${ }^{\circledR}$, ambos programas diseñados para jardines botánicos y otras colecciones científicas, o paquetes completos como ArcGIS ${ }^{\circledR}$ con una gran gama de aplicaciones; Biótica, programa manejado por la CONABIO, y programas comerciales como Excel ${ }^{\circledR}$ y Access ${ }^{\circledR}$, entre otros. En la actualidad, con el uso de microchips, los ejemplares valiosos pueden ser inventariados y revisados periódicamente, o en el caso de que se pierda la etiqueta de identificación, se recuperan sus datos fácilmente. La localización precisa de ejemplares en las áreas como arboretos y camellones se facilita por medio de una estación total electrónica u otros sistemas manuales de topografía.

\section{Conclusiones}

El factor más exitoso entre los jardines botánicos mexicanos es la educación ambiental, que se debe ampliar con 
más programas novedosos como la Fairchild Challenge, para tener aún más impacto sobre la sociedad. El financiamiento siempre ha sido un problema para los jardines botánicos, y se puede aliviar con la aplicación de la mercadotecnia para crear exhibiciones novedosas, eventos culturales y otros servicios para atraer más visitantes. La creación de centros de jardinería de alta calidad para venta de libros de horticultura, accesorios de jardinería, semillas y plantas, éstas últimas pueden provenir de las UMA y así contribuir al éxito de las mismas y la sustentabilidad. Los jardines botánicos mexicanos todavía tienen mucho que hacer para mejorar los labores de horticultura y curatoría de sus colecciones y debe ser de prioridad de cada jardín, que es esencial para su participación más eficiente en programas de investigación sistemática, restauración ecológica y contribución en el cumplimiento de las metas de la GSPC 2011-2020. México, uno de los países megadiversos del planeta debe tener como prioridad el desarrollo y apoyo de sus jardines botánicos como instituciones de investigación botánica, sin dejar a un lado el hecho de ser centros culturales y para el deleite del visitante que busca espacios de paz, tranquilidad y esparcimiento.

\section{Agradecimientos}

Los autores agradecen a la CONABIO, CONACYT y SEMARNAT, los cuales a través de varios proyectos y de bases de datos, investigación y apoyo a colecciones han contribuido en fortalecer los jardines botánicos mexicanos a lo largo de los años. Agradecemos a la Asociación Mexicana de Jardines Botánicos, A.C. por su labor incansable de mantener los jardines botánicos unidos y en comunicación. Los autores agradecen a Victoria Sosa, Roger Orellana y Javier Francisco Ortega por revisar con detalle al manuscrito y sus valiosas sugerencias para mejorarlo, y a Gina Gallo por revisar el manuscrito y sugerir mejoras en el estilo y redacción.

\section{Literatura citada}

BGCI. 2006. The Gran Canaria Declaration II: On Climate Change and Plant Conservation. Área de Medio Ambiente y Aguas del Cabildo de Gran Canaria, Jardín Botánico "Viera y Clavijo", Botanic gardens Conservation International, Richmond.

Blackmore S., Gibby M. y Rae D. 2011. Strengthening the scientific contribution of botanic gardens to the second phase of the Global Strategy for Plant Conservation. Botanical Journal of the Linnean Society 166:267-281.

Bramwell D. 1991. Botanic gardens in conservation: reintroduction into the wild. En: Heywood V.H. y Wyse Jackson P.S. Eds. Tropical Botanic Gardens: Their Role in Conservation and Development, pp. 209-216, Academic Press, Londres.

Bye R. 1994. Historia de los jardines botánicos: evolución de estilos, ideas y funciones. Revista Chapingo, Serie Horticultura 2:43-53.

Campbell P. 2006. The constant gardeners. Nature 440:845.
Choat B., Jansen S., Brodribb T.J., Cochard H., Delzon S., Bhaskar R., Bucci S.J., Feild T.S., Gleason S.M., Hacke U.G., Jacobsen A.L., Lens F., Maherali H., Martínez-Vilalta J., Mayr S., Mencuccini M., Mitchell P.J., Nardini A., Pittermann J., Pratt R.B., Sperry J.S., Westoby M., Wright I.J. y Zanne A.E. 2012. Global convergence in the vulnerability of forests to drought. Nature 491:752-756.

Cohen J.I., Williams J.T., Plucknett D.L. y Shands H. 1991. Ex situ conservation of plant genetic resources: global development and environmental concerns. Science 253:866-872.

Crane P.R., Hopper S.D., Raven P.H. y Stevenson D.W. 2009. Plant science research in botanic gardens. Trends in Plant Science 14:575-577.

Cuevas-Sánchez J.A. y Muñoz A.G. 2006. Conservación y mejoramiento in situ. En: SINAREFI Ed. Plan de Acción para la Conservación de los Recursos Fitogenéticos para la Alimentación y la Agricultura en México, pp. 2-17, Sistema Nacional de Recursos Fitogenéticos para la Alimentación y Agricultura, SAGARPA, México, D.F.

Cushing L., Kopas J.. 2011. Principal human rights impacts of climate change in Latin America. Reporte de la Asociación Interamericana para la Defensa del Ambiente (AIDA). San Francisco.

Da Silva J.M., Donaldson J.S., Reeves G. y Hedderson T.A. 2012. Population genetics and conservation of critically small cycad populations: a case study of the Albany Cycad, Encephalartos latifrons (Lehmann). Biological Journal of the Linnean Society 105:293-308.

De Ávila A., Bye R., Lascurain M., García A., Rodríguez M. y Chávez V. 2003. Declaración Oaxaca: Principios sobre el Acceso a los Recursos Genéticos y el Conocimiento Tradicional de la Flora Mexicana, y el Reparto de Beneficios Derivados de ellos en los Jardines Botánicos. Asociación Mexicana de Jardines Botánicos, A.C., México, D.F.

Durán D. 1967. Historia de las Indias de la Nueva España e Islas de la Tierra Firme. Editorial Porrúa, México, D.F.

García-Mendoza A.J. 2007. Los agaves de México. Ciencia 87:14-23.

Hardwick K.A., Fiedler P., Lee L.C., Pavlik B., Hobbs R.J., Aronson J., Bidartondo M., Black E., Coates D., Daws M.I., Dixon K., Elliott S., Ewing K., Gann G., Gibbons D., Gratzfeld J., Hamilton M., Hardman D., Harris J., Holmes P.M., Jones M., Mabberley D., Mackenzie A., Magdalena C., Marrs R., Milliken W., Mills A., Lughadha E.N., Ramsay M., Smith P., Taylor N., Trivedi C., Way M., Whaley O. y Hopper S.D. 2011. The role of botanic gardens in the science and practice of ecological restoration. Conservation Biology 25:265-275.

Herrera E., García-Mendoza A. y Linares E.1993. Directorio de los Jardines Botánicos de México. Asociación Mexicana de Jardines Botánicos, A.C., Publicación Especial 1. México, D.F.

Heslop-Harrison J. 1974. Genetic resource conservation: the end and the means. Journal of the Royal Society of Arts 122:157-169.

Heslop-Harrison J. 1976. Introduction. En: Simmons J.B., Beyer R.I., Brandham P.E., Lucas G.Ll. y Parry V.T.H. Eds. Conservation of Threatened Plants, pp. 3-7, Plenum Press, Nueva York.

Heywood V.H. 1991. Developing a strategy for germplasm conservation in botanic gardens. En: Heywood V.H. y Wyse Jackson P.S. Eds. Tropical Botanic Gardens: Their Role in Conservation and Development, pp. 11-23, Academic Press, Londres.

IB-UNAM. http://www.ibiologia.unam.mx/colecciones/frame.htm 
Kay J., Strader A.A., Murphy V., Nghiem-Phu L., Calonje M. y Griffith M.P. 2011. Palma corcho: A case study in botanic garden conservation horticulture and economics. HortTechnology 21:474-481.

Lascurain M. 2010. Quinientos años de jardines botánicos en México. En: Vovides A.P., Linares E. y Bye R. Jardines Botánicos de México: Historia y Perspectivas, pp. 61-77, Secretaría de Educación de Veracruz, Xalapa.

Lascurain R.M., Avendaño R.S., del Amo S., Niembro R.A., López B.C. y Trinidad R.E. 2010. Guía de Frutos Silvestres Comestibles en Veracruz. Fondo Sectorial para Investigación, el Desarrollo y la Innovación Tecnológica Forestal, Conafor, Conacyt, Xalapa.

Leadlay E. y Greene J. 1998. The Darwin Technical Manual for Botanic Gardens. Botanic Gardens Conservation International, Londres.

León-Portilla M. 1979. La Filosofía Náhuatl, Estudiada en sus Fuentes. Universidad Nacional Autónoma de México, Instituto de Investigaciones Históricas, México, D.F.

Linares E., Mazari M., Balcázar T., Bolaños R. y Bye R. 2006. Componentes esenciales en la planeación de un jardín botánico. En: Lascurain M., Gómez O., Sánchez O. y Hernández C.C. Eds. Jardines Botánicos: Conceptos, Operación y Manejo, pp. 35-53, Asociación Mexicana de Jardines Botánicos, A.C., Publicación Especial 5, México, D.F.

Luna V.E. 2006. Documentación y manejo de información de las colecciones de plantas vivas. En: Lascurain M., Gómez O., Sánchez O. y Hernández C.C. Eds. Jardines Botánicos: Conceptos, Operación y Manejo, pp. 113-118, Asociación Mexicana de Jardines Botánicos, A.C., Publicación Especial 5. México, D.F.

Marris E. 2006. Plant science: Gardens in full bloom. Nature 440:860-863.

Maunder M. 2006. El futuro de los jardines botánicos del trópico y la conservación de especies y hábitats. En: Lascurain M., Gómez O., Sánchez O. y Hernández C.C. Eds. Jardines Botánicos: Conceptos, Operación y Manejo, pp. 23-32, Asociación Mexicana de Jardines Botánicos, A.C., Publicación Especial 5. México, D.F.

Miranda J. 1960. Obras Completas Vol. I: Vida y Obra de Francisco Hernández. Instituto de Biología, Universidad Nacional Autónoma de México, México, D.F.

Molina-Moreno J.C. y Córdoba-Tellez L. 2006. Conservación ex situ. En: SINAREFI Ed. Plan de Acción Nacional para la Conservación de los Recursos Fitogenéticos para la Alimentación y la Agricultura en México, pp. 18-24, Sistema Nacional de Recursos Fitogenéticos para la Alimentación y la Agricultura en México, SAGARPA, México D.F.

Moore J.K. 1974. Botanic gardens and arboreta. En: Radford A.E., Dickison W.C., Massey J.R. y Bell C.R. Eds. Vascular Plant Systematics, pp. 775-790, Harper and Row, Nueva York.

NCCPG (National Council for the Conservation of Plants and Gardens). 2011. The National Plant Collections Directory 2011. National Council for the Conservation of Plants and Gardens, Londres.

Osborne R., Calonje M.A., Hill K.D., Stanberg L. y Stevenson D.W. 2012. The world list of cycads. En: Proceedings of the 8th International Conference on Cycad Biology (CYCAD 2008) Ed. Memoirs of the New York Botanical Garden 106:480-508.

Powledge F. 2011. The evolving role of botanical gardens. BioScience 61:743-749.
Prance G.T. 2010. A brief history of conservation at the Royal Botanic Gardens, Kew. Kew Bulletin 65:501-508.

Rae D. 2004. The role of horticulture in the global strategy for plant conservation. Sibbaldia 2:95-100.

Raven P.H. 1976. Ethics and attitudes. En: Simmons J.B., Beyer R.I., Brandham P.E., Lucas G.Ll. y Parry V.T.H. Eds. Conservation of Threatened Plants, pp. 155-179, Plenum Press. Nueva York.

REMIB.http://www.conabio.gob.mx/remib/doctos/remib_esp.html.

Rodríguez-Acosta M. 2000. Estrategia de Conservación para los Jardines Botánicos Mexicanos. Asociación Mexicana de Jardines Botánicos, A.C., México, D.F.

Rodríguez-Acosta M., Flores K.V. y Torres J.L.M. 2008. Especies prioritarias en el Jardín Botánico "Ignacio Rodríguez de Alconedo" de la Benemérita Universidad Autónoma de Puebla. Amaranto Nueva Epoca 1:19-23.

Rubluo A., Chávez V. y Martínez A. 1989. In vitro seed germination and reintroduction of Bletia urbana (Orchidaceae) in its natural habitat. Lindleyana 4:68-73.

Ruíz-Sánchez E., Sosa V. y Mejía-Saules M.T. 2011a. Molecular phylogenetics Mesoamerican bamboo Olmeca (Poaceae: Bambusoideae): Implications for taxonomy. Taxon 60:89-98.

Ruíz-Sánchez E., Sosa V., Mejía-Saules M.T., Londoño X. y Clark. L.G. 2011b. A taxonomic revision of Otatea (Poaceae: Bambusoideae: Bambuseae) including four new species. Systematic Botany 36:314-336.

SER (Society for Ecological Restoration). 2011. <http://www.ser. org/>(consultado 03 febrero 2012).

Sosa V. 2004. Colecciones botánicas. En: Fernández-Concha G.C., Sosa V., León de la Luz J.L. y Cortés J.L. Eds. Colecciones Biológicas, pp. 50-51, Consejo Nacional de Ciencia y Tecnología, Centro de Investigación Científica de Yucatán, México, D.F.

Sosa V., Mejía-Saules T., Cuéllar M.A. y Vovides A.P. 2013. DNA barcoding in endangered Mesoamerican groups of plants. The Botanical Review DOI 10.1007/S12229-013-9129-4.

Thuiller W., Lavorel S., Araújo M.B., Sykes M.T. y Prentice I.C. 2005. Climate change threats to plant diversity in Europe. Proceedings of the National Academy of Science USA 102:8245-8250.

University of Illinois. Malus domestica L.; http://urbanext.illinois. edu/apples/facts.cfm, consultado $1^{\circ}$ de noviembre de 2012

Valdés G.J. 1974. Los jardines botánicos. Revista de la Universidad Nacional Autónoma de México 29:11-16.

Vergara Silva F., Iglesias C. y Vovides A.P. 2002. La colección nacional de cícadas del Jardín Clavijero y la investigación de aspectos importantes de su biología. Biodiversitas 42:6-11.

Vovides A.P., Linares E. y Bye R. 2010b. Jardines Botánicos de México: Historia y Perspectiva. Secretaría de Educación de Veracruz, Xalapa.

Vovides A.P., Pérez-Farrera M.A. e Iglesias C. 2010a. Cycad propagation by rural nurseries in Mexico as an alternative conservation strategy: 20 years on. Kew Bulletin 65:603-611.

Vovides A.P., Iglesias C., Pérez-Farrera M.A., Vázquez Torres M. y Schippmann U. 2002. Peasant nurseries: A concept for an integrated conservation strategy for cycads in Mexico. En: Maunder M., Clubbe C., Hankamer C. y Groves M. Eds. Plant Conservation in the Tropics: Perspectives and Practice, pp. 421-444, Royal Botanic Gardens, Kew, Richmond.

Vovides A.P., González-Astorga J., Pérez-Farrera M.A., González D., Bárcenas C. e Iglesias C. 2007. The cycads of México: 25 
ANDRew P. Vovides ET AL.

years of research and conservation. En: Proceedings of Cycad 2005 The 7th International Conference on Cycad Biology Ed. Memoirs of the New York Botanical Garden 97:611-641.

Walther G.R., Post E., Convey P., Menzel A., Parmesan C., Beebee T.J.C., Fromentin J.M., Hoegh-Guldberg O. y Bairlein F.
2002. Ecological responses to recent climate change. Nature 416:389-395.

Wyse Jackson P.S. y Sutherland L.A. 2000. International Agenda for Botanic Gardens in Conservation. Botanic Gardens Conservation International, Richmond.

Recibido: 3 de diciembre de 2012

Aceptado: 6 de marzo de 2013 